ORIGINAL ARTICLE

\title{
The Scottish perinatal neuropathology study: clinicopathological correlation in early neonatal deaths
}

\author{
J C Becher, J E Bell, J W Keeling, N McIntosh, B Wyatt
}

Arch Dis Child Fetal Neonatal Ed 2004;89:F399-F407. doi: 10.1136/adc.2003.037606

The supplementary tables can be found at hittp:// adc.bmijournals.com/ supplemental/.

See end of article for authors' affiliations

....................

Correspondence to: Professor Mclntosh, Department of Child Life and Health, Reproductive and Developmental Medicine, 20 Sylvan Place, Edinburgh EH9 1UW, Scotland, UK; neil. mcintosh@ed.ac.uk

Accepted

4 November 2003
Background: A proportion of neonatal deaths from asphyxia have been shown to be associated with preexisting brain injury.

Objectives: (a) To compare the epidemiology of infants displaying signs of birth asphyxia with those not showing signs; (b) to examine the neuropathology and determine if possible the timing of brain insult comparing asphyxiated with non-asphyxiated infants; (c) to compare the clinical features of those born with birth asphyxia with and without pre-labour damage.

Methods: Over a two year period, all 22 Scottish delivery units collected clinical details on early neonatal deaths. Requests for post mortem included separate requests for detailed neuropathological examination of the brain. Infants were classified into two groups: birth asphyxia and non-birth asphyxia. Clinicopathological correlation was used to attempt to define the time of brain insult.

Results: Detailed clinical data were available on 137 of 174 early neonatal deaths that met the inclusion criteria. Seventy of 88 parents who had agreed to post mortem examination consented to a detailed examination of additional samples from the brain; in 53 of these cases the infant was born in an asphyxiated condition. All asphyxiated and encephalopathic infants, $38 \%$ of mature and $52 \%$ of preterm infants with features of birth asphyxia but without encephalopathy, and only one of 12 infants without any signs of birth asphyxia showed damage consistent with onset before the start of labour.

Conclusions: In a large proportion of neonatal deaths, brain injury predates the onset of labour. This is more common in infants born in an asphyxiated condition.
T he three major causes of neonatal death are lethal malformations, prematurity, and birth asphyxia. ${ }^{1}$ Whereas the general public considers major malformations and premature birth as unavoidable mischance, birth asphyxia implies a lack of care in labour. Although birth asphyxia is classically linked to intrapartum hypoxia-ischaemia in full term infants, often proceeding to a neonatal encephalopathythe so-called hypoxic-ischaemic encephalopathy, a proportion of preterm babies are also born in a neurologically depressed condition almost certainly related to poor oxygenation in labour. Asphyxia is acknowledged to be an imprecise term, but is still used regularly by the profession and parents. It may be implied by one or more of the following features: a low Apgar score $^{2-7}$; a baby who is difficult to resuscitate; metabolic acidosis in either the $\operatorname{cord}^{5689}$ or early neonatal blood samples; the development of neonatal encephalopathy. ${ }^{10-12}$ A history of these particular features may be sought retrospectively if an infant goes on to develop neurodevelopmental delay. None of these indicators, when applied prospectively to infants born in poor condition, has good sensitivity, specificity, or predictive value for neurodevelopmental delay or disability, although in full term infants the development of neonatal encephalopathy is more specific. ${ }^{13}$ It is clear that perinatal asphyxia is not likely to be an important factor in the development of every case of neonatal encephalopathy or in most cases of cerebral palsy. ${ }^{14-19}$ This view has been endorsed by a statement from the International CP Task Force. ${ }^{20} 21$

Obstetric care has seen dramatic changes over the last few decades. Most changes have contributed to the steadily falling stillbirth and neonatal death rates. ${ }^{12-25}$ However, despite better clinical care and widespread use of fetal monitoring and fetal blood sampling, full term infants continue to be born in a neurologically depressed condition. Such infants cause considerable distress to parents and staff. They contribute both to early neonatal mortality and to the pool of children who display later neurodevelopmental disability with cerebral palsy. Although in some cases obstetric risk factors can be identified, affected children also result from pregnancies and labours that, even when scrutinised critically, appear to be normal.

Litigation for perceived perinatal mismanagement is increasingly common, particularly in relation to infants born in a neurologically depressed condition-usually manifested by a poor Apgar score-and often reflexly labelled birth asphyxia. Some recent anecdotal reports and small series of infants born in poor condition have shown neuropathological abnormality at autopsy that must have preceded the onset of labour. These generally represent the collected experience of specialist referral centres ${ }^{26-30}$ or focus on a particular age group of infants-for example, those born preterm. ${ }^{31-35}$ Only rarely do the neuropathological studies include correlation with clinical factors. ${ }^{36-38}$ Further insights into cliniconeuropathological correlation in the first weeks of life are now being achieved by neuroimaging. ${ }^{39}$ This Scottish study was set up to identify neuropathological abnormalities in a population cohort of perinatal deaths and to explore the relation between clinical features and pathological findings. We report here the findings in the neonatal deaths.

The specific aims of this paper are to:

- review the epidemiology (sociodemographic, antenatal, and perinatal factors) of the early neonatal deaths overall 
and to compare infants who displayed signs of birth asphyxia with those who did not;

- investigate the neuropathological status in those infants in whom a post mortem was authorised, and to determine whether lesions could be of prenatal origin;

- determine if infants who have pre-existing brain damage are, when born alive, more likely to be born in an asphyxiated condition;

- compare the antepartum and intrapartum course of early neonatal deaths of infants born with birth asphyxia with and without pre-existing damage.

\section{METHODS}

\section{Study setting and patients}

The Scottish perinatal neuropathology study was a prospective observational and experimental study involving all 22 delivery units within Scotland. Patients were recruited during a two year period for each centre. The study started in January 1996, and recruitment of cases was completed by January 1999. The base study considered all perinatal deaths of infants who were $\geqslant 24$ weeks gestation at birth and $\leqslant 7$ days at time of death delivered in Scotland over the two year period. This paper concerns the epidemiology and neuropathology of the liveborn subset of the study cohort. The stillborn infants presented somewhat different features and will be reported on separately.

Infants with central nervous system or cardiac malformations, major chromosomal abnormalities, or central nervous system infection were excluded because it was felt that the neuropathological changes associated with such conditions might interfere with the interpretation of any changes superimposed by perinatal insult.

Figure 1 lists how the cohort of 692 qualifying perinatal deaths was reduced by various exclusions through the 221 liveborn infants to the 70 infants from whom the brain was available for examination in this study. These 70 infants were classified according to whether they displayed birth asphyxia (BA group) or not (noBA group). Analysis of those who died three days or less after the onset of labour allowed identification of pathological features likely to have predated labour and birth. Placentas were available for histological examination from 41 of the 70 infants.

\section{Ethical and consent procedures}

Before the start of the study, each delivery unit obtained approval from their local research ethics committee to approach appropriate parents. As different units received ethical permission at slightly varying times, the spread of data collection was three years, although it was two years for each individual centre. Cases were enrolled at the time of post mortem request by the clinician responsible for the care of the infant during life. A detailed clinical dataset was collected on all infants regardless of enrolment status. The purpose of the study was explained to parents. Signed consent was obtained for autopsy, and on a separate consent form, if authorised also for extended neuropathological research studies on the brain.

\section{Clinical details}

For each case a detailed questionnaire was completed by specially trained midwives or other local staff who recorded a battery of clinical information and the results of investigations relating to each pregnancy, labour, delivery, and neonatal course. This was entered into a central database (SPSS) by the study clinical coordinator (JCB). Information on the intrapartum cardiotocograph (CTG) was recorded if available.

\section{Diagnosis of asphyxio}

No test is available to accurately diagnose clinically important intrapartum asphyxia. The CTG is notorious for its poor predictive value..$^{40}{ }^{41}$ As one of the principle aims of the study was to determine if infants with pre-existing brain damage are predisposed to neurological depression at birth which might be labelled as birth asphyxia, we used fairly broad inclusion criteria.

- An Apgar score at five minutes of $\leqslant 5.0$; this is the traditional assessment and it is widely recognised that a low five minute Apgar score has an association, although weak, with both neonatal death and morbidity in surviving infants. ${ }^{42}$

- A cord or initial blood pH of < 7.1; obstetric epidemiology has shown that a scalp pH of less than 7.25 is abnormal and delivery is indicated if less than $7.2 .^{40}{ }^{41}$ The relation between scalp and cord $\mathrm{pH}$ is good with a sensitivity of $93 \%{ }^{43}$ However, the neonate is rarely difficult to resuscitate unless the cord $\mathrm{pH}$ is less than 7.0. We arbitrarily chose an intermediate level $(\mathrm{pH}<7.1)$ as indicating some degree of birth asphyxia in this group of early neonatal deaths. Recognising the limitations, we also used (in the absence of a cord $\mathrm{pH}$ ) a first blood gas with a $\mathrm{pH}$ less than 7.1 to indicate asphyxia.

- The presence of grade $2 / 3$ neonatal encephalopathy. This is widely accepted as having a closer association with significant birth asphyxia and long term neurodevelopmental disability. ${ }^{13}{ }^{44}$ The grading of encephalopathy used was that of Sarnat and Sarnat. ${ }^{45}$

Because of the diverse clinical circumstances, not all criteria were available for assessment in each case. Infants who displayed at least one of these criteria were classified as showing clinical evidence of birth asphyxia (BA group). If none of these criteria were present, the infant was included in the non-asphyxiated group (noBA).

\section{Pathological examination \\ Autopsies}

Autopsies were conducted in six Scottish centres, and the brain was retained in fixative for later examination. In the south east of Scotland, the fixed brains were examined in the Department of Neuropathology at the Western General Hospital, Edinburgh. Elsewhere they were sampled locally according to a previously agreed protocol. Up to 20 representative paraffin embedded blocks were prepared in each case from all areas of the cerebrum (including temporal hippocampus), and from the basal ganglia and thalami, midbrain, pons, medulla, vermis, and cerebellar hemispheres. These blocks were collected centrally for review and further investigation in Edinburgh. Paraffin sections were stained routinely with haematoxylin and eosin and luxol fast blue/ cresyl violet (myelin). Selected sections were investigated immunocytochemically for astrocytic status, using an antibody to glial fibrillary acidic protein and for microglia/ macrophages (antibodies to CD68 and MHCII) or stained with Perls Prussian blue stain (haemosiderin). The neuropathological appearances in grey and white matter were assessed independently in all cases by two observers (JEB and BW), who were initially blind to the clinical history. Selected cases were also reviewed by JWK. Recorded neuropathological features included neuronal eosinophilia and karyorrhexis, astrocytic hyperplasia, activated microglia and accumulation of macrophages, haemorrhage (recent and older), vascular responses, and foci of mineralisation and of infarction. The neuropathological features were then correlated with the gestational and postnatal age of the infant and 


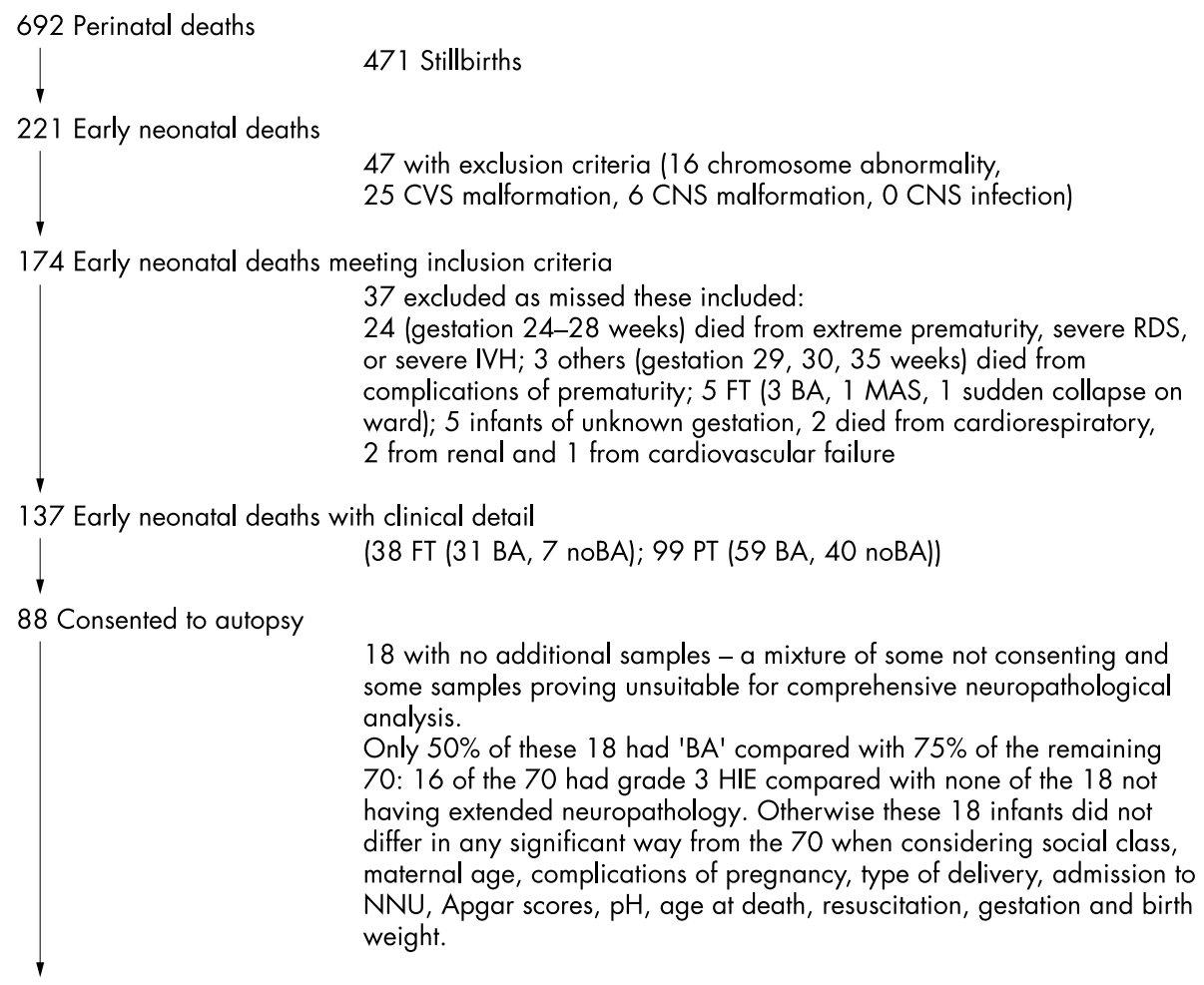

70 Consented to additional study samples

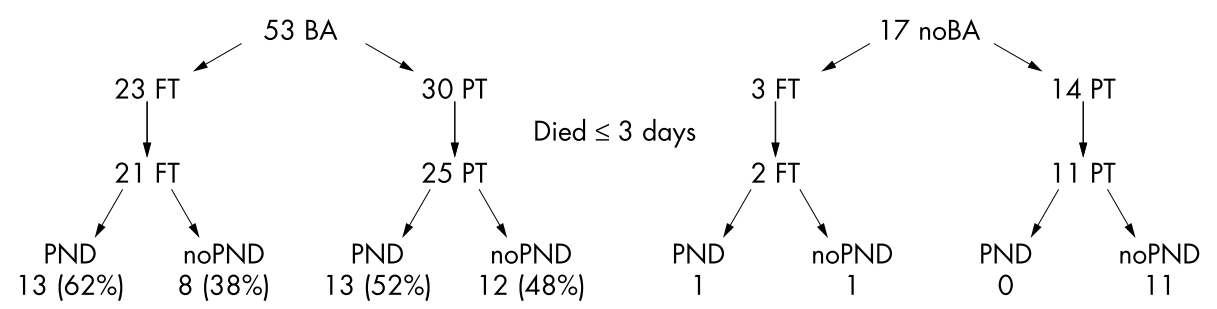

Figure 1 The Scottish perinatal deaths cohort. FT, full term; RDS, respiratory distress syndrome; PT, preterm $<37$ weeks; IVH, intraventricular haemorrhage; BA, birth asphyxia; MAS, meconium aspiration syndrome; noBA, no birth asphyxia; HIE, hypoxic-ischaemic encephalopathy; PND, prenatal brain damage; NNU, neonatal unit; noPND, no prenatal brain damage; CVS, cardiovascular system; CNS, central nervous system.

with the criteria of birth asphyxia, in combination and individually.

A judgment of whether the damage dated from before the onset of labour, and was therefore prenatal, was based in part on the presence of patently mature lesions such as established infarcts, previous haemorrhage, or extensive mineralisation. However, these features were present in the minority of brain damaged infants. More diffuse features such as definite macrophage infiltration/accumulation and/or prominent reactive astrocytic hyperplasia in white matter are thought to develop over a period of more than three days (table 1). We estimated that the presence or absence of prenatal brain damage could only be determined reliably in infants who died at $\leqslant 3$ days of age $(n=59)$.

\section{Placenta}

The placenta, cord, and membranes were examined macroscopically, and cord length, placental measurements, and trimmed weight were recorded. Any abnormality was described. Histological samples were taken to include a cross section of the umbilical cord, one strip of membranes (adjacent to the hole through which the baby was delivered, if identifiable), and two blocks of placenta with both fetal and maternal surfaces. Blocks and slides from the placenta and adnexa were submitted for central review in Edinburgh (JK). Histological evidence of infection, specifically chorioamnionitis in the extraplacental membranes or chorionic plate and funisitis, were recorded, as was villitis if generalised.

\section{Statistical analysis}

Data were recorded in SPSS. Descriptive statistics were used to examine the prevalence of clinical variables. The $\chi^{2}$ test with Yates correction (or Fisher's exact test where sample size was less than 20) was used to compare categorical variables, and the unpaired $t$ test or Mann-Whitney $\mathrm{U}$ test to compare the difference in continuous variables. Significance was assumed at $\mathrm{p}<0.05$, but we recognise that a large number of tests were performed, and some positive results at this level may have occurred by chance. As the epidemiology was performed on observational data, we leave the reader to consider the implications at this level rather than apply a correction such as that of Bonferroni. The statistical comparison of the pathology of asphyxiated and nonasphyxiated infants was made using $\chi^{2}$ tests with Yates' correction. 
Table 1 Timing of injury to the central nervous system after cerebral insult

\begin{tabular}{|c|c|c|}
\hline Pathological feature & $\begin{array}{l}\text { Timing of onset } \\
\text { after injury }\end{array}$ & References \\
\hline Neuronal eosinophilia & $6-24$ hours & $60-62$ \\
\hline Neuronal karyorrhexis & $12-48$ hours & $61,63-65$ \\
\hline Infarcts - necrosis & $3-8$ hours & $60,65,66$ \\
\hline Infarcts - cavitation & $14-42$ days & $65-68$ \\
\hline White matter gliosis & 3-11 days & $\begin{array}{l}31,32,58,60,61,65, \\
67,69,70\end{array}$ \\
\hline Grey matter gliosis & 3-5 days & $30,63,68,71$ \\
\hline Microglial upregulation & 3 hours -3 days & $60,61,66,67,71$ \\
\hline Macrophage infiltration & 3-7 days & $63,65-68$ \\
\hline Fresh haemorrhage & Minutes & 67 \\
\hline Haemosiderin deposits & 2-3 days & $27,67,72$ \\
\hline Mineralisation & 3-14 days & $58,60,65,67$ \\
\hline
\end{tabular}

\section{RESULTS}

\section{Population and study cohort}

Of the 692 deaths in the two years of the study, 221 were early neonatal deaths corresponding to the estimated early neonatal death rate of 2.5/1000 live births in Scotland.

Of the 137 deaths analysed (fig 1), 90 were classified as BA and 47 as noBA according to our liberal definition. Table 2 shows how they met the criteria for birth asphyxia. Most infants died from the effects of prematurity, congenital anomalies, or "anoxia". The causes of death included one case each of GMl gangliosidosis, laryngeal atresia, and diaphragmatic hernia, all of which may have contributed to the clinical picture of asphyxia. Twenty out of 137 (15\%) pregnancies studied were twin (19) or triplet (one). Complications of pregnancy were common, in particular, oligohydramnios $(20 \%)$, intrauterine growth restriction (14\%), premature rupture of membranes $(23 \%)$, and second or third trimester antepartum haemorrhage (29\%). Although abnormal serum screening for $\alpha$ fetoprotein and human choriogonadotrophin occurred in 14 pregnancies, in only five of these was amniocentesis carried out. The other six amniocenteses were performed for amnioreduction (five) or at maternal request (one). Of 62 cases of fetal anomaly scan, 21 were abnormal (including multiple abnormalities). Two infants were conceived following induction by ovulation stimulating drugs, and two by in vitro fertilisation. Emergency caesarean section took place in 57 (42\%) deliveries, of which 21 were performed before the onset of labour and 36 were intrapartum. Two infants were born by elective caesarean section, one because of a previous caesarean section and the other because it was a twin pregnancy. There was no excess over the expected proportion of early neonatal deaths delivered out of hours (2100-0900 and weekends; 61\%). An abnormal infection screen was found in 15 of the total group (group B Streptococcus (seven), coliforms (four), Staphylococcus aureus (two), others (two)), of which eight were thought to have died from overwhelming sepsis (three group B Streptococcus; one group A Streptococcus; two coliforms; one Pseudomonas, and one unidentified).

Seventy neonates were fully enrolled in this study with their parents agreeing to both an autopsy and the extended brain sampling. Of these 70, 53 were thought to be asphyxiated (BA group; 23 mature ( $\geqslant 37$ weeks) and 30 preterm (24-36 weeks) infants), and 17 did not appear to be asphyxiated (noBA group; three mature ( $\geqslant 37$ weeks) and 14 preterm (24-36 weeks) infants) (fig 1). The mature infants lived for between 15 minutes and seven days, with only three surviving for more than three days. The preterm infants lived for between five minutes and 6.8 days, with only eight infants surviving for more than three days. The ratio of asphyxiated (77\%) to non-asphyxiated (23\%) was slightly skewed in the group of autopsied infants towards asphyxiated cases when compared with the whole cohort of liveborn infants included in the detailed epidemiological survey ( $\mathrm{n}=137 ; 66 \%$ asphyxiated, $34 \%$ non-asphyxiated $)$.

\section{Clinical comparison of BA and noBA cohorts}

Detailed supplementary tables can be found at http:// adc.bmjjournals.com/supplemental/. Briefly, the mothers were comparable for age, weight, height, social class, marital status, parity, and all other factors examined (supplementary table 1). Mothers of infants who were born in an asphyxiated state were less likely to have received steroids during pregnancy $(20 \% \vee 36 \%, \mathrm{p}=0.036)$. Hyperemesis ( $8 \% \vee 23 \%$, $\mathrm{p}=0.013)$, placenta praevia $(2 \% \vee 11 \%, \mathrm{p}=0.037)$, intrauterine growth retardation $(10 \% \vee 23 \% \mathrm{p}=0.066)$, and pyrexia or flu-like illness during pregnancy $(6 \% \vee 17 \%$, $\mathrm{p}=0.061$ ) were less common in the BA cohort (supplementary table 2). Markers of fetal distress (supplementary table 3), such as meconium staining and cardiotocograph (CTG) abnormalities, ${ }^{40}$ were significantly more prevalent in the BA cohort $(26 \% v 11 \%, \mathrm{p}=0.040 ; 59 \% v 33 \%, \mathrm{p}=0.004)$. Intrapartum infection, indicated by positive vaginal swabs, maternal pyrexia, increased white cell count, or increased C reactive protein, occurred in 12 cases (Escherichia coli and other coliforms, group B Streptococcus, and Staphylococcus aureus) but was not more common in the BA group. Malpresentation was less common in the BA cohort $(30 \% v$ $45 \%, \mathrm{p}=0.087$ ).

The noBA cohort were of younger gestation $(29 v 32$, $p=0.017$ ), lighter, and had a smaller head circumference (supplementary table 4). The BA cohort, who had lower Apgar scores, required more resuscitation as a result. Eighteen $(20 \%)$ infants in the BA group were asystolic at birth. Infants in the BA cohort were more likely to die early compared with those in the noBA cohort (10.3 h $v 43 \mathrm{~h}$, $\mathrm{p}=0.002$ ). Of 137 infants, only 106 were admitted to a neonatal unit. Of the remaining 31 infants, five were born in good condition and died suddenly and unexpectedly: three were found dead in their cots on the postnatal ward after transfer from the labour ward, and two suffered a sudden acute deterioration in the labour ward after a normal delivery. Twenty four infants had severe birth asphyxia,

\begin{tabular}{|c|c|c|}
\hline Features of asphyxia & Full term & Preterm \\
\hline Total number of infants & 38 & 99 \\
\hline \multicolumn{3}{|l|}{ Single feature only } \\
\hline Apgar $\leqslant 5$ at $5 \mathrm{~min}$ & 9 & 35 \\
\hline Cord $\mathrm{pH}<7.1$ & 0 & 1 \\
\hline 1 st $\mathrm{pH}<7.1$ & 1 & 8 \\
\hline NNE & 1 & 0 \\
\hline \multicolumn{3}{|l|}{ Two features } \\
\hline Low Apgar and low pH & 7 & 9 \\
\hline Low Apgar and NNE & 2 & 1 \\
\hline Low $\mathrm{pH}$ and NNE & 0 & 1 \\
\hline \multicolumn{3}{|l|}{ Three features } \\
\hline Low $\mathrm{pH}$, low Apgar, and NNE & & \\
\hline $\begin{array}{l}\text { Total with some indication of } \\
\text { asphyxia }\end{array}$ & 31 (82\%) & $59(60 \%)$ \\
\hline \multicolumn{3}{|c|}{$\begin{array}{l}\text { All infants had a five minute Apgar score. Only } 12 \text { full term } \\
\text { infants and } 11 \text { preterm infants had cord pH measured. An } \\
\text { additional } 22 \text { full term infants and } 35 \text { preterm infants had the } \\
\text { pH measured on arrival in the local neonatal unit. } 16 \text { full } \\
\text { term infants at } 12 \text { hours of age were not paralysed, and } 14 \\
\text { of these had features of an encephalopathy. } 19 \text { preterm } \\
\text { infants remained alive and non-paralysed at } 12 \text { hours; six } \\
\text { had an encephalopathy. } \\
\text { NNE, Neonatal encephalopathy. }\end{array}$} \\
\hline
\end{tabular}


and, although they had signs of life at or shortly after birth, they could not be resuscitated sufficiently to move them to the neonatal unit. One extremely premature infant (a triplet) was given only compassionate care.

Clinical details of infants admitted to a neonatal unit were often limited because of early death (supplementary table 5). Within the first hour of birth, infants in the BA group had a considerably lower initial arterial blood $\mathrm{pH}$ (6.96 $v$ 7.25, $\mathrm{p}<0.001)$. In survivors of more than 12 hours, those in the BA group were more likely to have renal dysfunction $(55 \% \mathrm{v}$ $24 \%, \mathrm{p}=0.029)$ and to require assisted ventilation for poor respiratory drive $(33 \% \vee 3 \%, \mathrm{p}<0.001)$. The noBA cohort, in keeping with their shorter gestation, had a greater incidence of respiratory distress syndrome and were more likely to have received exogenous surfactant $(73 \% v 40 \%, \mathrm{p}=0.001)$ and to have muscular paralysis $(36 \% v 14 \%, p=0.011)$. In infants who survived for longer than 12 hours, abnormal neurology was documented in $83 \%$ of the BA cohort compared with $20 \%$ of the noBA cohort $(\mathrm{p}<0.001)$. Seizures were significantly more common (19\%) in the BA than in the no BA group (3\%) $(\mathrm{p}<0.027)$, but many infants $(\mathrm{n}=30)$ were treated prophylactically with anticonvulsants or paralysis, and many $(\mathrm{n}=61)$ died before seizures might have been expected. The groups were comparable for other features of systemic dysfunction, in particular coagulopathies, necrotising enterocolitis, cardiovascular instability, and glucose homoeostasis.

\section{Neuropathological findings and identification of prenatal brain damage}

Eighty eight infants underwent autopsy, and 70 parents authorised the additional samples required for this research study (fig 1). Table 3 shows the prevalence of neuropathological abnormalities in these 70 infants, classified into the BA and noBA groups (53 $v 17$ ) and according to their gestation (mature $v$ preterm). Table 4 shows similar data for the infants aged 3 days and less. A detailed table of clinicopathological correlation for each infant in the group of 27 with putative prenatal brain damage has been provided for the interested reader (supplementary table 6). In table 4, the BA group has been further subdivided according to whether encephalopathy was one of the features of birth asphyxia.

In both mature and preterm infants, the asphyxiated infants were more likely to show brain damage than the nonasphyxiated, although brain damage was not universally present in asphyxiated infants (tables 3 and 4). Some infants showed evidence of continuing brain damage, with recent events such as neuronal eosinophilia and fresh haemorrhage superimposed on older lesions including established infarcts, macrophage accumulation including cells laden with haemosiderin, extensive micromineralisation, and white matter gliosis. Infants with no evidence of asphyxia at birth (mostly preterm infants) were more likely than asphyxiated infants to appear virtually normal on neuropathological examination, and such changes as were present, including haemorrhage and neuronal eosinophilia, appeared to be recent except in two mature infants who displayed prominent gliosis.

In cases in which brain damage was present, a conclusion as to whether this was likely to be of prenatal origin could be achieved only in infants who died at $\leqslant 3$ days of age. This was based on the presence of abnormalities thought to first appear about three days after brain injury. There is no absolute certainty about the time needed for the different responses to become visible (table 1), but the presence of accumulations of macrophages and/or prominent astrocytic hyperplasia in human white or grey matter is generally assumed to require three days or more. Evidence from the literature for this timing is presented in more detail in supplementary table 7 . It is important to note that, of the 27 infants judged to have suffered prenatal brain damage, only four had survived for more than two days, six had survived one to two days, and all the rest (65\%) had survived for less than one day from the onset of labour. On this basis, 26 $(57 \%)$ of the asphyxiated group had evidence suggesting prenatal brain damage compared with one $(8 \%)$ of the nonasphyxiated group, a highly significant difference $(\mathrm{p}<0.005)$ (table 4).

Table 4 also shows that infants in the BA group who were encephalopathic displayed a particularly high prevalence of brain damage. Nine of 10 infants in this group showed macrophages or gliosis, or both, together with other confirmatory signs of continuing damage such as neuronal karyorrhexis and eosinophilia. Table 4 also highlights the fact that many of the brains of non-encephalopathic asphyxiated infants were apparently undamaged prenatally and that even by the time of death in the postnatal period, $31 \%$ of mature and $13 \%$ of preterm asphyxiated infants in this subgroup had apparently normal brains. Although the non-asphyxiated infants appeared to be more prone to postnatal or intrapartum damage, this difference was not significant $(p<0.059)$. Unsurprisingly, the preterm infants were more susceptible to damage of recent, and therefore probably, postnatal origin than were mature infants.

\section{Clinical factors associated with prenatal brain damage}

A careful comparison was made of the pregnancies leading to the births of infants with features of pre-labour damage (PND group, $\mathrm{n}=27$ ) compared with those without such damage (noPND group, $\mathrm{n}=32$ ). Fewer mothers in the PND group received antibiotics in pregnancy $(1 \vee 8, p=0.031)$, more had caesarean section (17 $v 10, \mathrm{p}=0.015)$ and emergency caesarean section (17 $v 9, \mathrm{p}=0.007)$ for CTG abnormalities ( $18 v 8, \mathrm{p}=0.005)$, and more had meconium present in the amniotic fluid ( $11 \vee 3, \mathrm{p}=0.005)$. The Apgar score was 0 at birth in $33 \%$ of the PND group, significantly more than in the noPND group ( $9 v 2, \mathrm{p}=0.008)$, and the former group were heavier and more mature $(2526 v 1824 \mathrm{~g}$, $\mathrm{p}=0.033$, and $34.6 \vee 31.2$ weeks gestation, $\mathrm{p}=0.051$ respectively). The PND group were more likely to be ventilated after birth for a poor respiratory drive $(8<3$, $\mathrm{p}=0.037)$, and, although both groups were acidotic, had a more acidic first $\mathrm{pH}(6.90 \vee 7.08, \mathrm{p}=0.022)$. The time to spontaneous respiration was longer ( $5 v 1$ minute, $\mathrm{p}=0.009$ ), and the five minute Apgar score was correspondingly less good $(2 \vee 5, \mathrm{p}=0.021)$. Reflecting the larger birth weight and more mature status, they had a higher first blood pressure (46v36 mm Hg, p = 0.019) and were less likely to receive surfactant $(4 v 13, \mathrm{p}=0.024)$. The time to death, however, was similar in the two groups ( $12 \vee 7$ hours, $\mathrm{p}=0.42)$.

No differences in sociodemographic or pregnancy factors were identified between the encephalopathic and nonencephalopathic asphyxiated groups, but CTG abnormalities were present in $80 \%$ of the former group and in only $43 \%$ of the latter group $(\mathrm{p}<0.04)$.

\section{Prenatal damage and the signs of birth asphyxia}

Table 5 shows the pathology of prenatal brain damage related to the criteria we used for birth asphyxia. Although the strongest clinical association with the features of pre-labour damage is the development of a neonatal encephalopathy after a low $\mathrm{pH}$ and a poor Apgar score at five minutes, it is of note that, of the 22 infants who had only a low Apgar score and then died and had a post mortem examination, 11 showed brain damage. By this evidence, a low Apgar score was the sole clinical indicator of prenatal damage in three of 
Table 3 Histological evidence of brain damage in 70 neonates

\begin{tabular}{|c|c|c|c|c|}
\hline \multirow[b]{2}{*}{ Pathological feature } & \multicolumn{2}{|c|}{ BA group ( $n=53$; asphyxiated infants) } & \multicolumn{2}{|c|}{$\begin{array}{l}\text { NoBA group ( } n=17 \text {; non-asphyxiated } \\
\text { infants) }\end{array}$} \\
\hline & Mature $(n=23)$ & Preterm $(n=30)$ & Mature $(n=3)$ & Preterm $(n=14)$ \\
\hline Neuronal eosinophilia & $14(61)$ & $9(30)$ & 2 & $5(36)$ \\
\hline Neuronal karyorrhexis & $11(48)$ & $8(27)$ & 0 & $0(0)$ \\
\hline Grey matter infarcts & $1(4)$ & $3(10)$ & 0 & $0(0)$ \\
\hline White matter gliosis & $11(48)$ & $14(47)$ & 2 & $0(0)$ \\
\hline Grey matter gliosis & $7(30)$ & $5(17)$ & 1 & $0(0)$ \\
\hline Microglial upregulation & 9 (39) & $14(47)$ & 1 & $1(7)$ \\
\hline Macrophages & $9(39)$ & $14(47)$ & 0 & $0(0)$ \\
\hline Fresh haemorrhage & $11(48)$ & $19(63)$ & 0 & $8(57)$ \\
\hline Haemosiderin deposits & $0(0)$ & $1(3)$ & 0 & $0(0)$ \\
\hline Mineral deposits & $2(9)$ & $8(27)$ & 2 & $1(7)$ \\
\hline
\end{tabular}

13 mature infants and in eight of 13 preterm infants. Only 16 mature and 19 preterm infants in the PND group survived to 12 hours and remained non-paralysed; of these, 14 mature and six preterm infants had an encephalopathy. Looked at another way, 14 full term infants had clinical neonatal encephalopathy. Eight of these had a post mortem examination, and all had evidence of prenatal damage. Only six preterm infants had neonatal encephalopathy. Two of these had a post mortem, and only one had evidence of prenatal damage.

\section{The placenta}

In 41 cases $(59 \%$ of those who had a post mortem examination), a placenta was available for examination. In seven cases, there was histological evidence of infection, and in 33 cases there was none. All of the seven infected placentas came from infants delivered prematurely. In two (25 and 27 weeks gestation), the inflammation was focal, and in four it was more generalised (at 24, 24, 30, and 35 weeks gestation). The placenta of an additional baby, born at 41 weeks gestation, showed focal acute deciduitis without inflammation of the placenta, membranes, or cord. Only two of these infants had evidence of prenatal brain damage. Thus there was virtually no concordance of placental and brain pathology.

\section{DISCUSSION}

\section{Early neonatal deaths}

A major aim of this study was to determine the neuropathology in a geographically defined cohort of early neonatal deaths and to seek associations with events in the mother's pregnancy, labour, and delivery, and with the infant's condition at birth and during the period before death. We excluded infants with chromosomal abnormalities and with abnormalities of the cardiovascular and central nervous systems because these might themselves lead to neuropathological changes. We were able to review 137 cases with carefully documented clinical detail, and 70 with extensive neuropathology.

\section{Birth asphyxia criteria}

Although intrapartum hypoxia manifesting as birth asphyxia is uncommon and in decline, ${ }^{46}$ it is still viewed as a potentially preventable cause of death or damage often with expensive medicolegal implications. Yet there is much evidence that neurodevelopmental delay and cerebral palsy are associated with birth asphyxia in only a minority of cases and also that most birth asphyxiated infants do not manifest developmental delay or cerebral palsy. ${ }^{47} 48$ We used broad inclusion criteria for the diagnosis of birth asphyxia to ensure that we missed no cases and were able to evaluate the individual clinical features of asphyxia in relation to neuropathological abnormality. This may have led to the inclusion of infants whose poor condition was due to other factors such as sepsis and/or metabolic disease, including case 10 (supplementary table 6) with gangliosidosis GMI. Two thirds of our cohort of 137 infants were born in a poor condition. We used the clinical finding of depression at birth manifested by Apgar scores or fetal/neonatal acidosis as a marker of an acute intrapartum event leading to birth asphyxia. An assessment for neonatal encephalopathy in

Table 4 Histological evidence of brain damage, including putative prenatal damage, in 59 neonates aged 3 days or less

\begin{tabular}{|c|c|c|c|c|c|c|}
\hline \multirow[b]{3}{*}{ Pathological feature } & \multirow{2}{*}{\multicolumn{2}{|c|}{$\begin{array}{l}\text { Encephalopathic } \\
\text { BA group ( } n=10 ; 17 \% \text { ) }\end{array}$}} & \multirow{2}{*}{\multicolumn{2}{|c|}{$\begin{array}{l}\text { No encephalopathy } \\
\text { BA group ( } n=36 ; 61 \% \text { ) }\end{array}$}} & \multirow{2}{*}{\multicolumn{2}{|c|}{$\begin{array}{l}\text { No encephalopathy } \\
\text { BA group ( } n=13 ; 22 \% \text { ) }\end{array}$}} \\
\hline & & & & & & \\
\hline & Mature $(\mathrm{n}=8)$ & Preterm $(n=2)$ & Mature $(n=13)$ & Preterm $(n=23)$ & Mature $(n=2)$ & Preterm $(n=11)$ \\
\hline Neuronal eosinophilia & $8(100)$ & 1 & $5(38)$ & $4(17)$ & 0 & $4(36)$ \\
\hline Neuronal karyorrhexis & $8(100)$ & 1 & $1(8)$ & $4(17)$ & 0 & 0 \\
\hline Grey matter infarcts & 0 & 0 & 0 & 0 & 0 & 0 \\
\hline White matter gliosis* & $7(88)$ & 1 & $4(31)$ & 9 (39) & 1 & 0 \\
\hline Grey matter gliosis* & $5(63)$ & 1 & 0 & $1(4)$ & 0 & 0 \\
\hline Microglial upregulation & $6(75)$ & 1 & $2(15)$ & 9 (39) & 0 & $1(9)$ \\
\hline Macrophages* & 7 (88) & 1 & $1(8)$ & $8(35)$ & 0 & 0 \\
\hline Fresh haemorrhage & $4(50)$ & 0 & $6(46)$ & $11(48)$ & 0 & $5(45)$ \\
\hline Haemosiderin deposits & 0 & 0 & 0 & 0 & 0 & 0 \\
\hline Mineral deposits & $1(13)$ & 0 & $1(8)$ & $6(26)$ & 1 & $1(9)$ \\
\hline *Estimated prenatal brain damage & $8(100)$ & 1 & $5(38)$ & $12(52)$ & 1 & 0 \\
\hline
\end{tabular}

Values in parentheses are percentages.

Mature, $\geqslant 37$ weeks; preterm, 24-36 weeks. 
Table 5 Features of asphyxia and presence of putative prenatal damage (PND)

\begin{tabular}{|c|c|c|c|c|c|c|}
\hline \multirow[b]{3}{*}{ Features of asphyxia } & \multicolumn{3}{|l|}{ Full term } & \multicolumn{3}{|l|}{ Preterm } \\
\hline & \multicolumn{2}{|l|}{ Total } & \multirow{2}{*}{$\frac{\text { PND }}{\text { at PM }}$} & \multicolumn{2}{|l|}{ Total } & \multirow{2}{*}{$\frac{\text { PND }}{\text { at PN }}$} \\
\hline & Clinical & PM & & Clinical & PM & \\
\hline \multicolumn{7}{|l|}{ Single feature only } \\
\hline Apgar $\leqslant 5$ at $5 \mathrm{~min}$ & 9 & 7 & 3 & 35 & 15 & 8 \\
\hline Cord $\mathrm{pH}<7.1$ & 0 & 0 & 0 & 1 & 0 & 0 \\
\hline 1 st $\mathrm{pH}<7.1$ & 1 & 1 & 1 & 8 & 5 & 2 \\
\hline NNE & 1 & 0 & 0 & 0 & 0 & 0 \\
\hline \multicolumn{7}{|l|}{ Two features } \\
\hline Apgar and low pH & 7 & 5 & 1 & 9 & 3 & 2 \\
\hline Apgar and NNE & 2 & 0 & 0 & 1 & 0 & 0 \\
\hline Low $\mathrm{pH}$ and NNE & 0 & 0 & 0 & 1 & 0 & 0 \\
\hline \multicolumn{7}{|l|}{ Three features } \\
\hline Low pH, low Apgar and NNE & 11 & 8 & 8 & 4 & 2 & 1 \\
\hline Total & 31 & 21 & 13 & 59 & 25 & 13 \\
\hline
\end{tabular}

combination with these markers would have been more specific, ${ }^{21}$ but many of our infants died within hours of delivery, and a record of neurological examination was not always obtained. In addition, the administration of muscle relaxants to a fifth of our population precluded such an assessment. Finally, 70\% of our group were preterm and thus unlikely to exhibit the classical signs of neonatal encephalopathy.

\section{Clinical}

The epidemiological background of this cohort is similar to other recent studies from the developed world. ${ }^{42}{ }^{49-51}$ Analysis of the maternal sociodemographic information and the detailed data from the pregnancy did not identify any reliable predictors for birth asphyxia or for neuropathological abnormalities. Significant placenta praevia and hyperemesis were protective against asphyxia in general, possibly because these mothers were more intensively monitored. Even taking neonatal encephalopathy in isolation as a marker for prenatal asphyxia, no differences were identified between encephalopathic and non-encephalopathic asphyxiated infants in the pregnancy or sociodemographic factors monitored in this study. A history of pyrexia or flu-like illness in pregnancy has previously been found to be associated with neonatal encephalopathy. ${ }^{51}$ Our series did not show this association, and pyrexia was more common in pregnancies that resulted in non-asphyxiated infants. Intrauterine growth restriction has previously been strongly associated with neonatal encephalopathy $y^{49} 52$ and affected 14\% of our population, although not just the asphyxiated infants.

CTG abnormalities are common and are poorly predictive of fetal acidosis. ${ }^{53}$ Both CTG abnormalities and meconium staining of liquor were more common in infants with prenatal damage in this study, and CTG abnormalities proved to be the only difference between the encephalopathic BA and non-encephalopathic BA groups ( $80 \% v 43 \%$, p < 0.04$)$. Randomised trials have shown that, although monitoring of fetal heart rate can reduce the numbers of neonatal seizures, there is no change in the incidence of long term neurological damage, ${ }^{54}$ suggesting that some fetal heart rate abnormalities may reflect prior compromise. Although meconium staining alone has a high false positive rate, ${ }^{55}$ it is associated with increased perinatal mortality and morbidity. ${ }^{56}$ It has been hypothesised that intra-amniotic meconium may cause vasoconstriction of the umbilical vessels ${ }^{57}$ inducing fetal hypoxia-ischaemia. This is difficult to substantiate after delivery.

\section{Neuropathology}

About half $(51 \%)$ of the 137 eligible infants had detailed neuropathological investigation. The range of neuropathological abnormalities resembles those reported in previous studies, ${ }^{28-35} 58606163-6973$ although the prevalence of neuronal damage and damage to the grey matter is higher than elsewhere. Judgments about neuropathological abnormalities are more difficult in the preterm than in the term brain. Despite these difficulties, comparison of the asphyxiated infants and those not apparently suffering from birth asphyxia shows clear differences in terms of neuropathological changes. Examination confined to infants who died within three days of the start of labour, and separation of the asphyxiated group into those with and without neonatal encephalopathy, identifies a spectrum of damage. Unsurprisingly, the mature infants who died after displaying neonatal encephalopathy are most likely to show neuropathological changes. All of the brains in this study were carefully examined to determine whether any damage could have occurred before the onset of labour. If it is accepted that features such as focal or diffuse astrocytic hyperplasia and parenchymal macrophage accumulation are cellular reactions that require three days to become established, we may conclude that most infants with features of birth asphyxia had sustained brain damage prenatally, including all eight of the full term encephalopathic group. We are unable to the establish the age of the damage, but the background of apparently normal brain development suggests that the insult was sustained not long before the start of labour. It is harder to draw conclusions about the preterm infants in this study, but the absence of neuropathological changes in virtually all the mature and most of the preterm infants who did not display asphyxia is reassuring.

We do not underestimate the difficulty of interpreting these neuropathological findings and attributing the time of onset. Every abnormality has been included in supplementary table 6 , whether focal or diffuse, recent or old, but we accept that interpretation is subjective. Establishing the timing is difficult because experimental studies are not possible in human infants, although classic studies were able to relate pathology findings to major clinical incidents. ${ }^{6067}$ Some experts also comment that the results of animal work may not be directly relevant to the human situation. ${ }^{6067}$ Immunocytochemical investigation is mandatory to separate reactive astrocytosis from myelination gliosis. A number of the classic studies were conducted before cell specific immunocytochemistry became available, although this is not the case for more recent papers. Earlier papers may not 
have always included, or added, the duration of labour as a factor in timing, and interpretation may be hampered by longer survival. It is noted that the infants with a history of encephalopathy had survived for more than one day in most instances. We concede that seizure activity may induce and accelerate some of the changes seen in the brains of such infants, but the presence of diffuse astrocytosis in other infants who had survived very few hours and who died with no evidence of seizure activity reinforces the possibility of prenatal origin. A more secure evidence base for timing neuropathological events awaits the evolution of new markers of cell damage and irreversible cell death. The clinical significance of some of the lesions described such as diffuse astrocytosis, and in particular their contribution to the cause of death, remains uncertain.

\section{Correlation of clinical factors and neuropathology}

This study has failed to identify any pointers that would predict the birth of a compromised infant. Abnormal CTG, and meconium staining of liquor were the only predictive factors for birth asphyxia or prenatal brain damage. Previous studies have reported an association between oligohydramnios and prenatal brain damage possibly related to impaired blood flow in the umbilical cord. Abnormal CTG was the only clinical factor differentiating the asphyxiated infants who displayed encephalopathy and neuropathological abnormality from those who did not. Recently the presence of prenatal infection has been linked to brain damage. We found no support for this association.

\section{Implications for surviving infants}

It is possible that the neuropathological findings reported here represent the most severe end of a spectrum of perinatal brain damage resulting in a fatal outcome, while infants surviving perinatal asphyxia might show lesser degrees of similar pathology. However, the possibility also exists that dead infants and survivors represent two completely different groups in terms of both causation and pathology. Recent neuroimaging studies of surviving neonates with encephalopathy, with or without seizures, have a bearing on these questions. A large study by Cowan et al ${ }^{39}$ concluded, on the basis of magnetic resonance imaging performed in the first two weeks of life, that brain damage in mature infants with neonatal encephalopathy was most often acute and of perinatal onset particularly in an encephalopathic group without seizures. Very few infants in that study displayed evidence of prenatal brain damage on magnetic resonance imaging. Neuropathological corroboration was achieved in very few cases. In the absence of immunocytochemical investigation of gliosis and brain macrophage accumulation in all deaths, their conclusions about the prevalence of prenatal abnormalities may be an underestimate. We have discussed the difficulty of timing the lesion in our own study, in which conclusions on the presence or absence of prenatal brain damage were confined to infants who died less than three days after the onset of labour and based on neuropathological examination rather than imaging. We suggest that the cerebral insult was probably sustained only shortly before the onset of labour (even possibly precipitating the onset of labour). Evidence of continuing neuronal damage was also present in our series, not dissimilar to the findings of Cowan et al, but this was often in addition to the damage identified as occurring before labour within the constraints of current knowledge. It might be expected that brain damage in survivors would be less extensive and severe than in those with a fatal outcome. Whether the brain damage observed in our study represents the result of persisting or repeated insult, or the onset of a potentially reversible cascade accruing from a single insult, is uncertain. A multistep pathological process might present opportunities for intervention to limit further brain damage.

The fact that a significant proportion of clinically asphyxiated infants display no evidence of brain damage, and that infants who are not asphyxiated at birth often display only recent postnatal damage, offers hope for a good clinical outcome if such infants could be identified and "rescued" by medical intervention. This study shows that the current battery of investigations associated with pregnancy and labour remain blunt instruments in accurately predicting the arrival of an asphyxiated and prenatally brain damaged infant. Future work must address the development of methods for detecting antepartum damage so that optimal management of these vulnerable fetuses can be planned. Further evidence is also required on evolution of cellular reactions in the developing brain. The findings in this study support the notion that the birth of a compromised "asphyxiated" encephalopathic infant is not necessarily the result of a mismanaged labour nor the lack of vigilance in pregnancy.

\section{ACKNOWLEDGEMENTS}

This study was funded by the Scottish Home and Health Department and by Wellbeing and was dependent on the collaboration of Scottish paediatric pathologists and neuropathologists: Drs N Alsanjari, D Doyle, I Graham, E S Gray, A G Howatson, S Lang, A M Lutfy, J McCullough, J MacKenzie, K J McKenzie, J L McPhie, E R Nairn, and $\mathrm{N}$ M Smith. We are also grateful to the midwives and medical staff in the 22 neonatal and obstetric units throughout Scotland whose enthusiasm and help in enrolling cases and collecting data made this study possible. We are also grateful for assistance with manuscript production by Ms Angela Penman. Finally we are indebted to the families who, despite the turmoil surrounding the deaths of their infants and knowing that the results would not personally help them at their time of grief, allowed these detailed investigations to proceed.

\section{Authors' affiliations}

J C Becher, N McIntosh, Department of Child Life and Health, University of Edinburgh, Edinburgh, Scotland, UK

J E Bell, B Wyatt, Department of Pathology, University of Edinburgh J W Keeling, Department of Paediatric Pathology, Royal Hospital for Sick Children, Edinburgh

\section{REFERENCES}

1 Confidential Enquiry into Stillbirths and Deaths in Infancy (CESDI). 8th Annual Report. Maternal and Child Health Consortium. 2001.

2 Sykes GS, Molloy PM, Johnson P, et al. Do Apgar scores indicate asphyxia? Lancet 1982:494-6.

3 Fields LM, Entman SS, Boehm FH. Correlation of the one-minute Apgar score and the $\mathrm{pH}$ value of umbilical arterial blood. South Med J 1983;76:1477-9.

4 Paneth N, Fox HE. The relationship of Apgar score to neurologic handicap: a survey of clinicians. Obstet Gynecol 1983;61:547-50.

5 Josten BE, Johnson TR, Nelson JP. Umbilical cord blood pH and Apgar scores as an index of neonatal health. Am J Obstet Gynecol 1987;157:843-8.

6 Ruth VJ, Raivio KO. Perinatal brain damage: predictive value of metabolic acidosis and the Apgar score. BMJ 1988;297:24-7.

7 Gilstrap LC, Leveno KJ, Burris JS, et al. Diagnosis of birth asphyxia on the basis of fetal $\mathrm{pH}$, Apgar score, and newborn cerebral dysfunction. Am J Obstet Gynecol 1989;161:825-30.

8 Amato M, Gambon RC, Von Muralt G. Accuracy of Apgar score and arterial cord-blood $\mathrm{pH}$ in diagnosis of perinatal brain-damage assessed by CK-BB isoenzyme measurement. J Perinat Med 1986;14:335-8.

9 Johnson JWC, Richards DS, Wagaman RA. The case for routine umbilical blood acid-base studies at delivery. Am J Obstet Gynecol 1990;162:621-5.

10 McShane M, Maguire S, McClure G, et al. Birth asphyxia, encephalopathy and outcome. Ir Med J 1987:80:421-2.

11 Niijima S, Levene MI. Post-asphyxial encephalopathy in a preterm infant. Dev Med Child Neurol 1989;31:395-7.

12 Nelson KB, Leviton A. How much of neonatal encephalopathy is due to birth asphyxia? Am J Dis Child 1991;145:1325-31.

13 Levene MI, Grindulus H, Sands C, et al. Comparison of two methods of predicting outcome in perinatal asphyxia. Lancet 1986:67-9.

14 Blair E, Stanley FJ. When can cerebral palsy be prevented? The generation of causal hypotheses by multivariate analysis of a case-control study. Pediatr Perinat Epidemiol 1993;7:272-301

15 Nelson KB. Relationship of intrapartum and delivery room events to long-term neurologic outcome [Review]. Clin Perinatol 1989;16:995-1007. 
16 Yudkin P, Johnson A, Clover LM, et al. Assessing the contribution of birth asphyxia to cerebral palsy in term singletons. Pediatr Perinat Epidemiol 1995;9:156-70.

17 Nelson KB, Leviton A. How much of neonatal encephalopathy is due to birth asphyxia? Am J Dis Child 1991; 145:1325-31.

18 Edwards AD, Nelson KB. Neonatal encephalopathies: time to reconsider the cause of encephalopathies. BMJ 1998;317:1537-8

19 Hall DMB. Birth asphyxia and cerebral palsy. BMJ 1989;299:279-82.

20 Bakketteig LS. Only a minor part of cerebral palsy cases begin in labour. BMJ 1999;319:1016-17

21 MacLennan A. A template for defining a causal relation between acute intrapartum events and cerebral palsy: international consensus statement. BMJ 1999:319:1054-9.

22 Confidential Enquiry into Stillbirths and Deaths in Infancy (CESDI). Annual Report for 1 January-December 1993. Part 1: summary of methods and main results. London: Department of Health, 1995.

23 Confidential Enquiry into Stillbirths and Deaths in Infancy (CESDI). 5th Annual Report. Maternal and Child Health Consortium, 1998.

24 Confidential Enquiry into Stillbirths and Deaths in Infancy (CESDI). 6th Annual Report. Maternal and Child Health Consortium, 1999.

25 Confidential Enquiry into Stillbirths and Deaths in Infancy (CESDI). 7th Annual Report. Maternal and Child Health Consortium, 2000.

26 Rorke LB. Pathology of perinatal brain injury. New York: Raven Press, 1982

27 Squier M, Keeling JW. The incidence of prenatal brain injury. Neuropathol Appl Neurobiol 1991;17:29-38.

28 Marin-Padilla $M$. Developmental neuropathology and the impact of perinatal brain damage. 1: haemorrhagic lesions in the neocortex. J Neuropathol Exp Neurol 1996;55:758-73.

29 Marin-Padilla M. Developmental neuropathology and the impact of perinatal brain damage. II: white matter lesions in the neocortex. J Neuropathol Exp Neurol 1997;56:219-35.

30 Marin-Padilla M. Developmental neuropathology and the impact of perinatal brain damage. III: Grey matter lesions in the neocortex. I Neuropathol Exp Neurol 1999:58:407-29.

31 Golden JA, Gilles FH, Rudelli RD, et al. Frequency of neuropathological abnormalities in very low birth weight infants. J Neuropathol Exp Neurol 1997;56:472-8.

32 Gilles FH, Leviton A, Jeffrey SM, et al. Groups of histopathologic abnormalities in brains of very low birth weight infants. J Neuropathol Exp Neurol 1998;57:1026-34.

33 Skullerud K, Westre B. Frequency and prognostic significance of germinal matrix hemorrhage, periventricular leucomalacia, and pontosubicular necrosis in preterm neonates. Acta Neuropathol 1986;70:257-61.

34 Leviton A, Paneth N. White matter damage in preterm newborns: an epidemiologic perspective. Early Hum Dev 1990;24:1-22.

35 Paneth N, Rudelli RD, Monte W, et al. White matter necrosis in very low birthweight infants: neuropathologic and ultrasonographic findings in infants surviving six days or longer. J Pediatr 1990;116:975-84.

36 Gaffney G, Squier M, Johnson A, et al. Clinical associations of prenatal ischaemic white matter injury. Arch Dis Child Fetal Neonatal Ed 1994;70:101-6.

37 Mito T, Kamei A, Takashima S, et al. Clinicopathological study of pontosubicular necrosis. Neuropediatrics 1993;24:204-7.

38 Ellis WG, Goetzman BA, Lindenberg JA. Neuropathologic documentation of prenatal brain damage. Am J Dis Child 1988;142:858-66.

39 Cowan FM, Rutherford MA, Groenendaal F, et al. Origin and timing of brain lesions in term infants with neonatal encephalopathy. Lancet 2003;361:736-42.

40 NICE. The use of electronic fetal monitoring; the use and interpretation of cardiotocography in intrapartum fetal surveillance. NICE, 2001.

41 Royal College of Obstetrics and Gynaecology. The use of elctronic fetal monitoring: the use and interpretation of cardiotocography in intrapartum fetal surveillance. RCOG, 2001

42 Gaffney G, Sellers S, Flavell V, et al. Case-control study of intrapartum care cerebral palsy, and perinatal death [see comments]. BMJ 1994:308:743-50.

43 Brandt-Niebelschutz S, Saling E. Indications for operative termination of labor on cardiotography and fetal blood analysis: the reliability of these methods. $J$ Perinat Med 1994;22:19-27.

44 Yudkin $\mathbf{P}$, Johnson A, Clover LM, et al. Clustering of perinatal markers of birth asphyxia and outcome at age five years. Br J Obstet Gynaecol 1994; 101:774-81.
45 Sarnat HB, Sarnat MS. Neonatal encephalopathy following fetal distress. Arch Neurol 1976;33:696-705.

$46 \mathrm{Kalter} \mathrm{H}$. Five decade international trends in the relation of perinatal mortality and congenital malformations: stillbirth and neonatal death compared. Int J Epidemiol 1991;20:173-9.

47 Blair E, Stanley FJ. Intrapartum asphyxia: a rare cause of cerebral palsy. J Pediatr 1988;112:515-19.

48 Nelson KB. What proportion of cerebral palsy is related to birth asphyxia? [editorial]. J Pediatr 1988;112:572-4.

49 Adamson SJ, Alessandri LM, Badawi N, et al. Predictors of neonatal encephalopathy in full term infants. BMJ 1995;311:598-602.

50 Badawi N, Kurinczuk JJ, Keogh JM, et al. Intrapartum risk factors for newborn encephalopathy: the Western Australia case-control study. BMJ 1998;317:1554-8.

51 Badawi N, Kurinczuk JJ, Keogh JM, et al. Antepartum risk factors for newborn encephalopathy: the Western Australia case-control study. BMJ 1998;317:1549-53

52 Gunn AJ, Gunn TR. Changes in risk factors for hypoxic-ischaemic seizures in term infants. Aust NZ J Obstet Gynaecol 1997;37:36.

53 Steer PJ, Eigbe F, Lissauer TJ, et al. Interrelationships among abnormal cardiotocograms in labour, meconium staining of the amniotic fluid, arteria cord blood pH and apgar scores. Obstet Gynecol 1989;74:715-21.

54 MacDonald D, Grant A, Sheridan-Pereira M, et al. The Dublin randomised controlled trial of intrapartum fetal heart rate monitoring. Am J Obstet Gynecol 1985;152:524-39.

55 Nelson KB, Grether JK. Potentially asphyxiating conditions and spastic cerebral palsy in infants of normal birth weight. Am J Obstet Gynecol 1998; 152:524-39.

56 Berkus $M$, Langer $O$, Samueloff $A$, et al. Meconium-stained amniotic fluid: increased risk for adverse neonatal outcome. Obstet Gynecol 1994;84: 115-20.

57 Altshuler G. Some placental considerations related to neurodevelopmental and other disorders. J Child Neurol 1993;8:78-94.

58 Gilles FH, Murphy SF. Perinatal telencephalic leucoencephalopathy. J Neurol Neurosurg Psychiatry 1969;32:404-13.

59 Rorke LB. Anatomical features of the developing brain implicated in pathogenesis of hypoxic-ischaemic injury. Brain Pathol 1992;2:211-21.

60 Norman MG. Perinatal brain damage. Perspect Pediatr Pathol 1978;4:41-92.

61 Low JA, Robertson DM, Simpson LL. Temporal relationships of neuropathologic conditions caused by perinatal asphyxia. Am J Obstet Gynecol 1989;160:608-14

62 Graeber MB, Blakemore WF, Kreutzberg GW. Cellular pathology of the central nervous system. In: Graham DI, Lantos PL, eds. Greenfield's neuropathology. London: Arnold, 2002:123-9.

63 Friede RL. Ponto-subicular lesions in perinatal anoxia. Arch Pathol 1972:94:343-54.

64 Wigglesworth JS, Bridger JE. Neuropathological clues to the timing of early brain lesions. In: Lou HC, Griesen G, Larsen JF, eds. Brain lesions in the newborn. Alfred Benzon Symposium 37. Copenhagen: Munksgaard, 1994: 165-73.

65 Squier MV. Acquired diseases of the nervous system. In: Keeling JW, ed. Fetal and neonatal pathology. London: Springer Verlag, 2001:633-58.

66 Banker BQ. The neuropathological effects of anoxia and hypoglycemia in the newborn. Dev Med Child Neurol 1967;9:544-50.

67 Ellis WG Goetzman BW, Lindenberg JA. Neuropathologic documentation of prenatal brain damage. Am J Dis Child 1988;142:858-66.

68 Kinney HC, Armstrong DD. Perinatal neuropathology. In Graham DI Lantos PL, eds. Greenfield's neuropathology. London: Arnold, 2002:519-606

69 Roessmann U, Gambetti P. Pathological reaction of astrocytes in perinatal brain injury. Immunohistochemical study. Acta Neuropathol 1986;70:302-7.

70 Norenberg MD. Astrocyte responses to CNS injury. J Neuropathol Exp Neurol 1994;53:213-20.

71 Del Bigio MR, Becker LE. Microglial aggregation in the dentate gyrus: a marker of mild hypoxic-ischaemic brain insult in human infants. Neuropathol Appl Neurobiol 1994;20:144-51.

72 Vanezis P. Bruising: concepts of ageing and interpretation. In: Rutty GN, ed. Essentials of autopsy practice. London: Springer-Verlag, 2001:221-40.

73 Norman MG. Antenatal neuronal loss and gliosis of the reticular formation thalamus, and hypothalamus. A report of three cases. Neurology 1972;22:910-16. 


\section{PostScript}

\section{LETTERS}

\section{Pain relief during common neonatal procedures: a survey}

We conducted a survey of neonatal pain relief practices for common procedures across the United Kingdom as a baseline for improving our own practice, and we here present the results.

We sent a questionnaire to all Scottish hospitals, and units from the rest of the United Kingdom if they had 40 or more maternity beds $(\mathrm{n}=96) ;{ }^{1}$ the response rate was $85 \%$. Analgesia was used in $82 \%$ of units for elective intubation, the commonest agent used being morphine $(79 \%)$, followed by fentanyl. Analgesia was also used in $11 \%$ of the units for intravenous cannulation and in $10 \%$ for heelpricks. The analgesia most commonly used for cannulation was sucrose or dextrose. Some $5 \%$ of units stated that they used morphine for radial arterial lines but these infants were already ventilated and receiving morphine.

These data appear to give a snapshot of current practice, but we cannot know how far unit guidelines translated into the actual experience of the babies. As pain in the neonatal period has immediate and long term consequences, ${ }^{12}$ and preterm infants may be exposed to many painful procedures during their hospital stay, there is some way to go before we can claim that neonates are getting optimum pain control. ${ }^{12}$ The wider use of sucrose and topical anaesthetics (if safety concerns can be adequately addressed) seem likely to be the quickest routes to improving the situation.

\section{Acknowledgements}

We thank all the neonatal units who participated in this survey.

C Rennix, C M Manjunatha, S E Ibhanesebhor

Correspondence to: Neonatal Unit, Wishaw General Hospital, Wishaw ML2 ODP, Lanarkshire, Scotland, UK; Chikka10@hotmail.com

doi: 10.1136/adc.2003.045351

\section{References}

1 Foster GL. Good birth guide, 1st ed. Perth, Australia: Vermillion, 2002.

2 Chiswick ML. Assessment of pain in neonates [commentary]. Lancet 2000;355:6-8.

\section{US bioethics fall short of world standards}

In their study of motion resistant pulse oximetry in neonates, Sahni et $a l^{1}$ obtained approval from their institutional review board and consent from the parents of the infants involved. Nevertheless, the study fails the most basic principles of bioethics, and this calls into question the value of institutional review boards and points to a yawning chasm between American ethical practices and world ethical standards.
The recognised criteria for ethical experimentation are the Nuremberg Code $(1947)^{2}$ and the Declaration of Helsinki (1964) as amended. ${ }^{3}$ The Nuremberg Code requires the consent of the subject, which obviously could not be obtained in this case. The Declaration of Helsinki provides for the consent of the legal representatives of minor children in certain limited instances:

"For a research subject who is legally incompetent, physically or mentally incapable of giving consent or is a legally incompetent minor, the investigator must obtain informed consent from the legally authorized representative in accordance with applicable law. These groups should not be included in research unless the research is necessary to promote the health of the population represented and this research cannot instead be performed on legally competent persons. ${ }^{\prime 3}$

This provision is inapplicable in this instance because this research did not promote the health of the population group represented and because this research easily could have been performed on legally competent adults.

Male neonatal non-therapeutic circumcision violates basic human rights to security of the person and to freedom from torture, inhuman, or degrading procedures. A recent study found that neonatal circumcision fails all ethical tests. ${ }^{4}$ Moreover, the Norwegian Council for Medical Ethics advised the Norwegian Medical Association that the circumcision of boys is not consistent with important principles of medical ethics, has no established medical benefit, and causes pain even with the use of local anaesthesia. ${ }^{5}$ Nontherapeutic circumcision of children violates articles 1,2, and 20 of the European Convention on Human Rights and Biomedicine. ${ }^{6}$

The institutional review board must be more than a rubber stamp to approve whatever is proposed. Clearly, world ethical standards were not considered in this instance. It is time for American bioethics boards and committees to adopt world standards.

J V Geisheker, G Hill Doctors Opposing Circumcision, Suite 42, 2442 NW Market Street, Seattle Washington 98107, USA; iconbuster@earhlink.net

doi: $10.1136 /$ adc. 2003.047373

\section{References}

1 Sahni R, Gupta A, Ohira-Kist K, et al. Motion resistant pulse oximetry in neonates. Archives of Disease in Childhood Fetal and Neonatal Edition 2003;88:F505. Available at: http:// adc.bmijournals.com/cgi/content/full/ fetalneonatal $\% 3 \mathrm{~b} 88 / 6 / \mathrm{F} 505$

2 The Nuremberg Code. BMJ 1996;313:1448, Available at: http://bmi.bmijournals.com/cgi/ content/full/313/7070/1448.
3 Declaration of Helsinki, Adopted by the 18th World Medical Assembly, Helsinki, Finland, June 1964, and later amended. Available at: http:// www.wma.net/e/policy/b3.htm.

4 Hodges FM, Svoboda JS, Van Howe RS. Prophylactic interventions on children: balancing human rights with public health. J Med Ethics 2002;28:10-16, Available at: http:// jme.bmijournals.com/cgi/content/abstract/28/ $1 / 10$.

5 Gulbrandsen P. Rituell omskiæring av gutter. Tidsskr Nor Lægeforen 2001;121:2994, Available at: http://www.tidsskriftet.no/pls/lts/ pa_lt.visSeksjon?vp_SEKS_ID $=419642$.

6 European Convention on Human Rights and Biomedicine (1997). Adopted at Oviedo, 4 April 1997. Available at: http://conventions.coe.int/ Treaty/en/Treaties/Html/164.htm.

\section{Time is of the essence}

Accurate time keeping is of great import in all resuscitation settings and none more so than in the medicolegal minefield of perinatology. Recent cases have shown accurate documentation is of vital importance, especially the recording of times. Two studies have delineated considerable discrepancies in clock times in patient care settings ${ }^{12}$; these may exacerbate any medicolegal issues. A prospective observational study assessed clock accuracy in paediatric and neonatal resuscitation areas in a hospital with three sites, separated by up to 18 miles. Senior paediatric cover was provided by one middle grade and one consultant, making accuracy of timing extremely important. The accuracy of the consultants' watches was prospectively assessed without warning.

A total of 39 clock times were taken and compared with the true time as per the speaking clock. In total, the mean (SD) difference was - 11 (40) seconds. The labour suite clocks had a mean of $+6(20)$ seconds. The neonatal unit clocks had a mean of -109 (107) seconds. When paediatric resuscitation areas throughout the Trust were compared, the mean was +18 (43) seconds. The consultants' watches had a mean of +8 (54) seconds.

In the maternity hospital, the labour suite clocks have been changed to "satellite controlled" ones costing five pounds ninety nine pence. This has resulted in their close correlation, with obvious medicolegal benefits. The other clinical areas studied did not have this technology in place, but the results are considerably better than in previous studies. The accuracy of the consultants' watches was exceptional, as no warning was given. This is a reminder to all to document times accurately and evidence enough that resuscitation areas in hospitals should use modern technology and have a centrally controlled time system to avoid needless errors in annotation. We leave you to take your own time to decide.

A Crow, M Davidson, J P McClure

Correspondence to: Paediatric Department, Crosshouse Hospital, Kilmarnock, Scotland, UK; markgdavidson@doctors.org.uk

doi: 10.1136/adc. 2003.048512 


\section{References}

1 Wong CM, Stenson BJ, Laing AL. As time goes by. Scot Med J 2002;47:138-9.

2 Lerner EB, Billitier AJ 4th, Adolf JE. Ambulance, fire, and police dispatch times compared with the atomic clock. Prehosp Emerg Care 2000:4:28-30.

\section{Atrial flutter in preterm babies}

Atrial flutter is uncommon in neonates without congenital heart disease or cardiac surgery. It forms about $3 \%$ of cardiac arrhythmias in the newborn. ${ }^{1}$ Although idiopathic atrial flutter can occur in the fetus, ${ }^{2}$ accounting for $30 \%$ of fetal arrhythmias in one series, ${ }^{4}$ spontaneous conversion often occurs during birth. I share our experience of two preterm babies who had atrial flutter associated with maternal opiate abuse. There are no previous case reports on this association.

The first case was of a 27 week gestation baby born to a mother with mild cerebral palsy who was abusing drugs such as heroin, crack cocaine, and alcohol and was on a methadone programme during pregnancy. The baby was ventilated from birth for hyaline membrane disease. He had withdrawal symptoms from day 2 in spite of a maintenance infusion of diamorphine, which was then gradually increased. On day 3 , he suddenly developed one brief narrow complex tachycardia followed by a similar persistent tachycardia. This was initially diagnosed as supraventricular tachycardia, and he received appropriate treatment with no effect. On review by a cardiologist, atrial flutter was confirmed. Echocardiography ruled out any structural heart disease. The atrial flutter lasted for seven hours. The heart finally reverted to a sinus rhythm with a second dose of digoxin. The baby continued to receive a maintenance dose of digoxin. There was no recurrence of the atrial flutter.

The second case is of a 28 week preterm baby born to a mother who was a heroin addict and was on a methadone programme during the last trimester of pregnancy. The baby developed hyaline membrane disease and was initially managed with head box oxygen and then nasal continuous positive airways pressure. From day 2 he needed ventilation (with diamorphine maintenance) He developed withdrawal symptoms and later, two episodes of atrial flutter (fig 1).

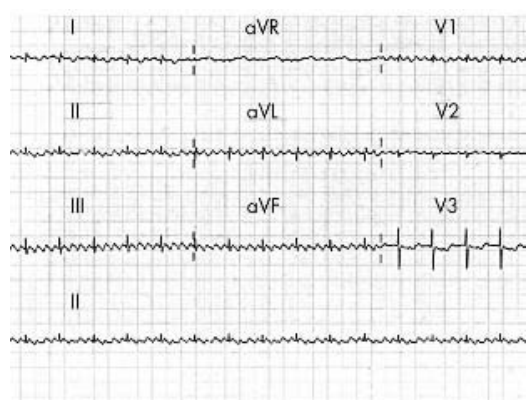

Figure 1 Electrocardiogram showing classical saw toothed flutter waves.
Diamorphine was increased to control the withdrawal symptoms. He spontaneously reverted to sinus rhythm and had no further episodes of atrial flutter. No structural heart disease was found on echocardiography.

These episodes of atrial flutter clearly happened in conjunction with other symptoms of opiate withdrawal. Sympathetic excitation is known to occur during opiate withdrawal. We do not know if this predisposes preterm babies, in whom atrial excitation occurs more readily, to this type of arrhythmia. Until we have further case reports, we will not be certain about this association, and the occurrence of this arrhythmia in these cases may be coincidental. However, we know that this type of arrhythmia, if persistent, can be serious, and immediate treatment will be life saving. Hence preterm babies should be monitored closely during opiate withdrawal.

S Sivakumar

Neonatal Unit, Birmingham Women's Hospital, Edgbaston, Birmingham B15 2TL, UK; s_siva_kumar@yahoo.com

doi: 10.1136/adc. 2003.046474

\section{References}

1 Southall DP, Johnson AM, Shinebourne EA, et al. Frequency and outcome of disorders of cardiac rhythm and conduction in a population of newborn infants. Pediatrics 1981;68:58-66.

2 Lisowski LA, Verheijen PM, Benatar AA, et al. Atrial flutter in the perinatal age group: diagnosis, management and outcome. $J$ Am Coll Cardiol 2000;35:771-7.

3 Naumburg E, Reisenfield T, Axelsson O. Fetal tachycardia: intrauterine and postnatal course. Fetal Diagn Ther 1997; 12:205-9.

4 Jaeggi E, Fouron JC, Drblik SP. Fetal atrial flutter diagnosis: clinical features, treatment, and outcome. J Pediatr 1998;132:335-9.

\section{Is mesenteric blood flow compromised during phototherapy in preterm neonates?}

We have previously reported that abdominal distension, visible "ropy" bowel loops, and bile stained gastric aspirates (manifestations of ileus) without loose watery stools are more often observed in preterm neonates having conventional phototherapy (CPT) than in those not having this treatment. ${ }^{1}$ Reported changes in the mesenteric blood flow as well as peripheral blood flow and cardiac output during CPT indicate that mesenteric ischaemia may occur during CPT in preterm neonates. ${ }^{2-4}$ We hypothesised that mesenteric blood flow may be compromised during CPT in preterm neonates who are not being fed. If our hypothesis was true, mesenteric ischaemia may explain ileus during CPT in preterm neonates.

In a prospective observational study, superior mesenteric artery blood flow (maximum, minimum) velocity and resistive index (RI) were measured by ultrasound pulsed Doppler in 14 consecutive preterm neonates before and 8-12 hours after the start of CPT. At the time of the study, they did not have associated common risk factors for ileus such as patent ductus arteriosus, indomethacin, sepsis, electrolyte imbalance, and enteral feeds. Their ventilatory/oxygen needs were minimal, and cardiovascular support was not required. The birth weight, gestational age, and postnatal age of the enrolled neonates were 885-1410 g, 27-29 weeks, and 2-4 days respectively. The mean (SD) maximum velocity (VMAX) and RI before and after the start of CPT were not significantly different: VMAX, $0.41(0.13) \vee 0.50(0.11) \mathrm{m} / \mathrm{s}(\mathrm{p}=0.10) ; \mathrm{RI}$, $0.73(0.08) \quad v \quad 0.70 \quad(0.08) \quad(\mathrm{p}=0.10)$. Minimum velocity after CPT was, however, significantly increased: $0.06(0.04) v 0.16$ $(0.05) \mathrm{m} / \mathrm{s}(\mathrm{p}<0.001)$. Ileus developed 4.8 (2.1) days after the initiation of CPT in 8/14 neonates despite the absence of the risk factors studied.

Increased superior mesenteric artery end diastolic blood flow velocity may indicate photorelaxation of the mesenteric vascular smooth muscle during CPT. ${ }^{5}$ CPT per se may be a risk factor for ileus in preterm neonates.

R Kadalraja, S K Patole, R Muller, J S Whitehall Department of Neonatology, Kirwan Hospital for Women, and School of Public Health and Tropical Medicine, James Cook University, Queensland,

Australia

Correspondence to: S Patole, Department of Neonatal Paediatrics, KEM Hospital for Women, University of Western Australia, Perth, Western Australia 6008; skpatole@hotmail.com doi: $10.1136 /$ adc. 2004.057646

\section{References}

1 Kadalraja R, Thomas E, Patole SK, et al. Is phototherapy a risk factor for ileus in high-risk neonates? Proceedings of Pediatric Academic Societies Annual Meeting, Baltimore May 2001. Pediatr Res 2001:120A.

2 Pezzati $M$, Biagiotti $R$, Vangi $V$, et al. Changes in mesenteric blood flow response to feeding: conventional versus fiber-optic phototherapy. Pediatrics 2000; 105:350-3.

3 Wu PYK, Wong WH, Hodgman JE, et al. Changes in blood flow in the skin and muscle with phototherapy. Pediatr Res 1974;8:257-62.

4 Benders MJ, Van Bel F, Van de Bor M. Cardiac output and ductal reopening during phototherapy in preterm infants. Acta Paediatr 1999:88:1014-19.

5 Furchgott RF. Endothelium-dependent relaxation, endothelium derived relaxation factor and photorelaxation of blood vessels. Semin Perinatol 1991;15:11-15.

\section{CORRECTION}

doi: 10.1136/adc.2002.037606corr 1

J C Becher, J E Bell, J W Keeling, et al. The Scottish perinatal neuropathology study: clinicopathological correlation in early neonatal deaths (Arch Dis Child Fetal Neonatal Ed 2003:89:F399-407). In Table 4, the second row, last column heading was published incorrectly and should read: NoBA group $(n=13 ; 22 \%)$. We apologise for this error. 